\title{
CALIDAD DE SERVICIO PERCIBIDA POR LOS USUARIOS DE LOS PROGRAMAS DE POSTGRADO EN GERENCIA DE LA FACULTAD DE CIENCIAS ECONÓMICASY SOCIALES
}

\author{
Por: Dianela Zuleta ${ }^{1}$, Caterina Clemenza ${ }^{2}$ y Rubén Araujo $^{3}$
}

\section{RESUMEN}

El artículo es producto de una investigación cuyo objetivo fue analizar calidad de servicio percibida por los usuarios de los Programas de Postgrado en Gerencia de la Facultad de Ciencias Económicas y Sociales (FCES-LUZ) de la Universidad del Zulia. La investigación es de tipo descriptivo con diseño no experimental, transaccional y de campo. La población estuvo conformada por estudiantes activos, de diferentes programas de postgrado ofrecidos por FCES-LUZ. El tamaño de la muestra se determinó a través de muestreo aleatorio simple con asignación proporcional. El instrumento de recolección de datos estuvo constituido por un cuestionario estructurado, contentivo de la variable que se estudió, basado en el modelo SERVQUAL. El mismo fue validado por expertos. La confiabilidad se determinó a través del coeficiente de Alpha Cronbach. Los resultados indican que el usuario califica el servicio como poco satisfactorio, observándose debilidades en la ejecución de los servicios complementarios a los procesos de enseñanza, y donde el indicador seguridad personal revela los niveles de insatisfacción más elevados. Se recomienda la creación y puesta en práctica de campañas orientadas al desarrollo de una cultura de servicio, centradas en atender las expectativas y necesidades del usuario.

Palabras clave: Calidad de servicio, Satisfacción del usuario, Estudios de postgrado, Universidad

Clasificación JEL: 123, M10, M31

1. Economista. Magister en Gerencia de Empresas. Mención Gerencia de Mercadeo. División de Estudios para Graduados de la Facultad de Ciencias Económicas y Sociales de la Universidad del Zulia. e-mail: dianelazuleta@gmail.com

2. Doctora en Ciencias Gerenciales. Post doctora en Ciencias de la Educación Profesora. Investigadora Titular adscrita al Instituto de Investigaciones de la Facultad de Ciencias Económicas y Sociales (FCES) de la Universidad del Zulia (LUZ). Acreditada al Programa de Estímulo al Investigador e Innovador (PEII) ONCTI. e-mail: caterinaclemenza@yahoo.es

3. Doctor en Ciencias Gerenciales. Magister en Gerencia de Empresas. Economista, Comunicador Social. Profesor-Investigador Asociado. Jefe del Departamento de Ciencias Humanas del Núcleo Costa Oriental del Lago de la Universidad del Zulia Acreditado Programa de Estímulo al Investigador e Innovador (PEII) ONCTI. e-mail raraujove@yahoo.es

Fecha de recepción: 2 de marzo 2014 Fecha de aprobación definitiva: 28 de marzo de 2014 


\title{
SERVICE QUALITY PERCEIVED BY USERS OF THE POST GRADE MANAGEMENT PROGRAMS AT THE ECONOMICS AND SOCIAL SCIENCES FACULTY
}

\author{
By: Dianela Zuleta, Caterina Clemenza y Rubén Araujo
}

\begin{abstract}
The article is the result of a research aimed to analyze the quality of service perceived by users of the Post Grade Management Programs , of Economics and Social Sciences Faculty (FCES - LUZ ), University of Zulia. The research is descriptive, not experimental, transactional and field design. The population consisted of active students from different graduate programs offered by FCES - LUZ. The sample size was determined using simple random sampling with proportional allocation. The data collection instrument was composed for an structured questionnaire, content of the variable being studied, based on the SERVQUAL model. The instrument was validated by experts. The reliability was determined using Cronbach Alpha coefficient. The results indicate that users call the service unsatisfactory, showing weaknesses in the implementation of the supplementary teaching processes services, and where the personal safety indicator shows higher levels of dissatisfaction. A campaign on the creation and implementation of a development-oriented service culture, focused on meeting the expectations and needs of the user is recommended.
\end{abstract}

Keywords: Service quality, user satisfaction, postgraduate studies, University.

JEL Classification: 123, M10, M31. 


\section{INTRODUCCIÓN}

En el mundo de hoy, el desarrollo económico ha dejado de tener como base la disposición de abundantes recursos naturales y mano de obra barata, y sí en el fortalecimiento del conocimiento y de las innovaciones tecnológicas, es decir, que el desarrollo económico está cada vez más asociado a la habilidad de un país para adquirir y aplicar conocimientos técnicos y socioeconómicos, los cuales se enfocan en el incremento de la producción de bienes y servicios que van a beneficiar a la sociedad. Hasta ahora, existen empresas que enfocan más su atención en la adquisición de maquinaria que en la preparación y buena remuneración de sus empleados, sin tomar en cuenta la influencia que esto pueda tener en la prestación del servicio.

Hoy en día, los directivos de las organizaciones a nivel mundial han incrementado su interés por desarrollar el marketing de servicios, a través del cual se pueden orientar para que sus organizaciones sobrevivan en el mercado, ya que no es solo cuestión de enfocar la atención en el control de los precios, la producción, entre otros, sino que también en el servicio que se le presta al cliente. Para ello, se debe tomar en cuenta la atención al personal que labora en la organización, sobretodo en aquel que tiene contacto directo con el cliente, en el sentido de que se le debe brindar un buen trato, un clima laboral agradable, así como también una buena remuneración acorde con su preparación profesional y cargo que ocupe, entrenamiento profesional, para que el empleado se encuentre contento con la organización y así la represente bien ante sus clientes, brindándoles un buen servicio.

Una persona, antes de ir a una empresa a comprar un producto o servicio, espera recibir un servicio de buena calidad como por ejemplo, recibir un trato amable por parte de los empleados, recibir respuestas ante sus dudas de manera eficiente, eficaz y oportuna, rodearse de un ambiente físico cómodo y agradable, entre otras cosas. Para ello, la empresa debe tener conocimiento sobre las necesidades tanto de sus clientes activos como potenciales para tratar de cubrir esas necesidades. Un servicio de buena calidad comprende la disminución de la brecha entre las expectativas y percepciones de los clientes, es decir, que mientras la percepción del servicio prestado supere a las expectativas del cliente, mayor será la fidelidad de este hacia la organización.

Cuando una organización logra superar las expectativas de buen servicio a sus clientes, estos quedarán satisfechos y puede lograr su fidelidad a ella. También contará con la recomendación de este cliente a otras personas, lo que permitirá que más clientes se acerquen a la empresa. Esto se logra a través de la calidad de servicio, la cual es una evaluación dirigida que refleja las percepciones del cliente sobre ciertas dimensiones específicas del servicio: confiabilidad, responsabilidad, seguridad, empatía y tangibles (Zeitahlm y Bitner, 2002). La calidad de servicios es uno de los componentes del marketing de servicios, el cual es aplicable a cualquier tipo de organización, es decir, tanto en las que producen bienes como en las que producen servicios, entre ellas se encuentran las instituciones educativas, en las cuales se debería poner en práctica esta rama del marketing para poder ofrecer un buen servicio a sus clientes (estudiantes). 
Una de las organizaciones que ofrecen un servicio que es considerado muy importante por la sociedad, es la universidad, que dentro del Sistema Educativo es llamado Sector Universitario, cuya razón de ser es la formación de profesionales, dotándolos de los medios necesarios para que una vez que sean incorporados en el mercado laboral, puedan poner en práctica sus conocimiento en las organizaciones, tanto públicas como privadas, donde desempeñen su carrera profesional, con el fin de contribuir al progreso de las organizaciones y por ende, al desarrollo de un país.

Debido a lo explicado en el párrafo anterior, las autoridades gubernamentales deben tomar muy en cuenta entre sus planes, el desarrollo del sector universitario, haciendo que éste ofrezca una buena calidad en el servicio prestado desde el punto de vista académico, administrativo, infraestructura, seguridad, entre otros. Para prestar un buen servicio debe haber una buena comunicación entre los sectores público, universitario y privado para determinar cuáles son los perfiles profesionales que se requieren en el mercado laboral y así, estos puedan ser incluidos en los programas académicos de las universidades.

A través de los estudios de cuarto nivel, los profesionales pueden profundizar o complementar sus conocimientos obtenidos en pregrado y/o en su experiencia laboral, para ponerlos en práctica en las empresas donde trabajan. Es por eso que las autoridades universitarias deben preocuparse por ofrecer un servicio de buena calidad a sus clientes (estudiantes) tanto desde el punto de vista académico como de otros servicios.

Una vez que las universidades han detectado cuáles son las necesidades de las empresas en cuanto al adiestramiento de su personal, deben promocionar a la sociedad los programas referentes a estudios de cuarto nivel que ofrecen, para que esta tome las decisiones adecuadas con respecto a la utilización de los servicios prestados.

Es por ello que a través de esta investigación se analizó la calidad de servicio percibida por los usuarios de los Programas de Postgrado en Gerencia de la Facultad de Ciencias Económicas y Sociales, por medio de la cual se le aportará a la institución información sobre cómo están funcionando sus servicios, es decir, cuáles son las expectativas y la percepción que tienen sus clientes (estudiantes) al respecto, para que sus directivos puedan tomar las medidas pertinentes.

\section{CONSIDERACIONES METODOLÓGICAS}

Esta investigación es descriptiva, con diseño no experimental, transaccional y de campo. La población está constituida por los estudiantes activos para el primer semestre del año 2012 de los programas de Gerencia de Empresas, Gerencia Pública, Especialidad en Gerencia de Empresas Turísticas y Doctorado de Ciencias Sociales: Mención Gerencia, de la División de Estudios para Graduados de la Facultad de Ciencias Económicas y Sociales de la Universidad del Zulia. 
Siendo la distribución de la población de 964 estudiantes de Gerencia de Empresas, 67 de Gerencia Pública, 28 de la Especialidad en Gerencia de Empresas Turísticas y 39 en el Doctorado de Ciencias Sociales: Mención Gerencia. Se aplicó un muestreo aleatorio estratificado, donde los estratos están representados por los tres programas de postgrado. En la investigación no existen indicios de algún valor conocido de $p$. Por lo tanto, se asume un valor hipotético de $p$ igual 0.50 , el cual ofrece un valor más conservador posible para la varianza de una proporción. Considerando un error máximo admisible e igual a 0,05 y un nivel de confianza asumido de $90 \%$, se tiene un valor de z igual $1.645 \mathrm{y}$ un tamaño de muestra $n$ igual a 271. El tamaño de la muestra es determinado para cada estrato. Se considera la asignación proporcional, dividiendo la muestra total en partes proporcionales al tamaño de la población de cada estrato.

Los datos fueron recolectados durante 5 días hábiles consecutivos, para disminuir la posibilidad que las respuestas fuesen sensibles a alteraciones puntuales en el servicio. Se aplicó el instrumento a los participantes que asisten a sus actividades académicas entre un horario de clases comprendido entre las 6:30 p.m. y las 8:30 p.m.; el centro de recolección de la información se ubicó en cada salón de clases, previa autorización del profesor.

El instrumento de recolección de los datos primarios está constituido por un cuestionario, conformado por una sección tipo tradicional y una sección tipo escala. La sección tipo tradicional del cuestionario es empleada para evaluar los objetivos específicos relativos a las dimensiones, datos socioeconómicos y motivación. Esta parte está conformada por siete ítems que reflejan los datos socioeconómicos de los maestrantes, así como los motivos para seleccionar los programas de postgrado.

Mientras que la sección tipo escala del cuestionario es representada por el instrumento SERVQUAL, aplicado para evaluar los objetivos específicos relativos a las expectativas y percepción de los usuarios y estuvo constituido por veintinueve aseveraciones.

Las expectativas se evaluaron, de acuerdo al grado de importancia que el cliente le confirió a cada afirmación según la siguiente escala: (1) Nada Importante, (2) Poco Importante, (3) Medianamente Importante, (4) Importante y (5) Muy Importante. Mientras que las percepciones se evaluaron, de acuerdo al grado de cumplimiento que el cliente le confirió a la División de Estudios para Graduados de la Facultad de Ciencias Económicas y Sociales de la Universidad del Zulia, con respecto a cada afirmación para lo cual selecciona una alternativa de la siguiente escala: (1) Totalmente en desacuerdo, (2) En desacuerdo, (3) Medianamente de acuerdo, (4) De acuerdo y (5) Totalmente de acuerdo.

La validación del instrumento de recolección de datos fue definida mediante la revisión por parte de un grupo conformado por cinco (5) expertos, mientras que para la confiabilidad se aplicó el coeficiente Alpha de Cronbach a una prueba piloto dirigida a veinte (20) estudiantes seleccionados por asignación proporcional de acuerdo a la población de cada estrato. Se determinó un coeficiente de confiabilidad igual a 0,94 , por lo cual se considera confiable el instrumento de recolección de datos. 


\section{ALGUNOS ASPECTOS TEÓRICOS}

\section{Calidad de la Educación Superior en Venezuela}

En Venezuela, existen cuatro niveles educativos formados por: la educación inicial, educación primaria, educación media y universitaria, esta última es el objeto de interés para esta ponencia. La base de la educación superior está formada por los niveles de pregrado y postgrado. El nivel de pregrado es el encargado de la formación profesional del individuo, la cual le ayuda a contribuir en el desarrollo de las empresas de los diferentes sectores de la economía a través de los conocimientos adquiridos en el mencionado nivel de instrucción. El nivel de postgrado, es el encargado de suministrarle conocimientos al individuo profesional y que les sirve de complemento o profundización de los conocimientos adquiridos en pregrado, lo cual le ayuda a escalar posiciones en las empresas.

La Constitución Nacional vigente, en efecto, establece en su Artículo 2 que: "la educación y el trabajo son los procesos fundamentales" para alcanzar los fines de la sociedad y del Estado. Los Artículos 102 y 103 declaran la educación como un "derecho humano y un deber social" al cual todos los ciudadanos pueden tener acceso en igualdad de condiciones de calidad, oportunidad y gratuidad (hasta el pregrado universitario), sin más limitaciones que las derivadas de sus aptitudes, vocación y aspiraciones.

El Artículo 109, por su parte, declara, con relación a las universidades, que el Estado "reconoce la autonomía universitaria como principio y jerarquía que permite a la comunidad académica dedicarse a la búsqueda del conocimiento a través de la investigación para beneficio de la Nación”. En este sentido, las universidades tienen autonomía para darse sus normas de gobierno, planificar, organizar, elaborar y actualizar los programas de investigación, docencia y extensión y para la administración eficiente de su patrimonio, bajo el control que, a tales efectos, establezca la ley.

De esta manera se establece por una parte, la autonomía universitaria y por la otra el control y vigilancia que el Estado debe ejercer para garantizar la calidad y pertinencia social de las actividades docentes, de investigación y extensión y la eficiencia en la gestión de los recursos humanos, materiales y financieros que el Estado otorga a las instituciones de educación superior.

De acuerdo a Morles y otros (2002), la educación superior venezolana a través de su historia pero, sobre todo, en su evolución reciente, se ha caracterizado por un crecimiento acelerado, una gran heterogeneidad y desarticulación institucional y una gran preocupación por la postergación de soluciones a los problemas de pertinencia, calidad, financiamiento y vacíos jurídicos de esta actividad.

Las universidades son autónomas, sus autoridades establecen las reglas desde todo punto de vista, pero siempre respetando la Ley. Sin embargo, el Estado vigila cómo va evolucionando la calidad de la educación en las universidades, la eficiencia del personal que labora en ellas, así como también la administración de los recursos financieros y materiales, que el Estado les asigna. 
Desde la década de los setenta, se han aplicado diversas iniciativas de evaluación de las instituciones de educación superior, pero la falta de seguimiento y otros factores presentes en el diseño, planificación y ejecución de tales iniciativas, no permitió el avance necesario para hacer de estas experiencias un proceso permanente. No obstante, conviene destacar cuatro de esas experiencias:

$\checkmark$ El sistema establecido por el CNU desde 1975, cumpliendo con mandatos legales, mediante el cual todo proyecto de creación de programas o instituciones de educación superior debe someterse a una evaluación por parte de la Oficina de Planificación del Sector Universitario (OPSU), organismo que posee una Unidad especializada.

$\checkmark$ La experiencia practicada por el Consejo Nacional de Investigaciones Científicas y Tecnológicas (CONICIT, hoy FONACIT), organismo que en 1976 creó un Programa de Financiamiento de Postgrados nacionales, el cual implica la evaluación de los cursos aspirantes a tal financiamiento, y que en 1991 creó el Sistema de Promoción al Investigador (SPI) con el cual se reconoce y estimula económicamente a los investigadores universitarios o no, que se destaquen en sus tareas de investigación.

$\checkmark$ Las Jornadas de Evaluación de los Programas de Postgrado de la Universidad Central de Venezuela, las cuales se llevan a cabo con cierta regularidad desde 1981 en tres niveles (programas, facultades y central), las cuales han permitido a esta casa de estudios mantener un debate permanente sobre los problemas de la educación avanzada.

$\checkmark \quad$ El sistema nacional de acreditación de programas de postgrado que desde 1987 lleva a cabo el Consejo Consultivo Nacional de Estudios de Postgrado, el cual permite que hoy se pueda hablar en Venezuela de programas de postgrado acreditados (es decir, que satisfacen unos criterios mínimos de calidad y han aceptado una evaluación externa) y programas no acreditados, cuya calidad puede ser cuestionada.

Esfuerzos adicionales de prácticas evaluativas se han concretado desde la OPSU, realizando evaluaciones y diagnósticos en diversas áreas de su competencia tales como; admisión y selección de estudiantes; clasificación de las universidades experimentales; oferta institucional y demanda estudiantil; planta física; estructura de financiamiento y otros.

La Oficina de Planificación del Sector Universitario (OPSU), se ha encargado concretamente en realizar evaluaciones y diagnósticos relacionados con el área que le concierne como: admisión y selección de estudiantes, clasificación de las universidades experimentales, oferta institucional y demanda estudiantil, planta física, estructura de financiamiento y otros.

En 1993, en el Consejo Nacional de Universidades (CNU), se realizó un taller sobre nuevas instituciones y programas universitarios, en el cual se llegó a la conclusión de que se necesita establecer un sistema nacional de evaluación institucional de la educación superior, llegándose a concretar ésta en el año 2001 con la creación de dicho sistema por el CNU por resolución número 383 del 26/01/2001 y el inicio de su puesta en ejecución. 
El sistema propuesto se fundamentó conceptualmente en tres elementos: la calidad universitaria, la evaluación como proceso de control y aseguramiento de la calidad universitaria y la evaluación como acreditación. En este sistema también sobresale un diseño que permite asegurar la calidad de las instituciones universitarias en tres fases: creación de instituciones y programas, seguimiento o supervisión y rendición de cuentas. En cuanto a la acreditación, se contó con un mecanismo a través del cual se pudo reconocer y certificar los niveles de excelencia de las instituciones y programas en sus tres fases: autoevaluación, evaluación por pares y certificación, abarca a todas las universidades del país en sus diferentes niveles y modalidades (presencial, a distancia y virtual) y las funciones de docencia, investigación y extensión (OPSU 2001).

El personal docente universitario ha dado la suficiente importancia para que el subsistema tenga un buen funcionamiento, debido a que la matrícula estudiantil tuvo un gran incremento en la década de los años setenta, y esto conllevó a la incorporación de un gran número de profesores universitarios sin la capacitación y experiencia que se requería. Por lo tanto, se concluye que la capacidad pedagógica de un docente es la que determina la calidad de la educación superior del individuo.

A pesar de que la Ley exige que el profesor universitario tenga una buena formación pedagógica, en la legislación no se señalan los sistemas adecuados para lograr esa buena formación pedagógica. Es por ello que la Universidad Central de Venezuela, entre otras instituciones, en 1974 creó programas que pueden mejorar esta condición, como lo es el Sistema de Actualización Docente del Profesorado (SADPRO), el cual ha atendido a un gran número de profesores.

En Venezuela, las personas tienen claridad de la existencia de estos procesos y también de la existencia de aquellos factores que le permiten participar de manera provechosa en esa serie de cambios que están ocurriendo a nivel mundial. Como es bien sabido, este país es altamente dependiente del exterior y de su renta petrolera. Romper esa circunstancia pasa, necesariamente por el fomento de la creatividad, las capacidades y trabajo tesonero de sus políticos, científicos y técnicos, cuyo éxito depende a la vez de su desarrollo profesional, su educación de postgrado, de su educación continua y permanente, todos a partir de una posición ideológica que corresponda con el mayor interés nacional (Azuaje, 2007).

\section{Satisfacción del Cliente}

Según Albrecht y Zenke (1998), el logro y el mantenimiento de un nivel de servicio superior que ofrezca cualquier organización o institución se inicia de la comprensión y el contacto con los usuarios. La calidad del servicio que conducirá a esfuerzos autónomos por parte de los empleados a fin de mejorar y mantener la calidad de servicio por sus propios medios, ellos aceptan la excelencia del servicio y a la vez son responsables de la enorme satisfacción del usuario, pues el usuario en este caso marca su nivel de satisfacción cuando vuelve por más servicio ya que él decide si el servicio ha cubierto sus necesidades.

De la misma manera Muller (1999), expone que la calidad del servicio tiene que superar las expectativas de los usuarios, ya que la calidad de servicio una vez que 
el usuario lo ha recibido, es igual al servicio, que él había esperado. Estos aspectos son percibidos por el empleado, y es este quien debe mantener el servicio actualizado, estudiar los aspectos negativos, y tomar medidas correctivas esenciales para la mejora del servicio, sin duda el usuario detectará por alguna expresión o queja cualquier insatisfacción del servicio, o sea, que no cubra sus expectativas, el cual la mayoría de los casos determina si es conveniente.

Por otra parte de acuerdo con Shaw (1997), sostiene que la satisfacción es la respuesta de sociedad del cliente, es un juicio cerca de un rango del producto o servicio, en sí mismo, proporcionando un nivel placentero de recompensa que se relaciona con el servicio.

Zeithaml y Bitner (2002), interpretan la satisfacción como la evaluación que realiza el cliente respecto del servicio, en término de si el servicio responde a sus necesidades y expectativas. Se presume que al fracasar los cumplimientos de las necesidades y las expectativas, el resultado que se obtiene es la insatisfacción con dicho servicio.

La satisfacción del cliente es influida por las características específicas del producto o servicio y las percepciones de la calidad. También actúan sobre la satisfacción las respuestas emocionales de los clientes, lo mismo que las atribuciones y sus percepciones de equidad, a definir:

- Características del producto y del servicio. Los clientes de los servicios, por lo general, realizan intercambios entre las diversas características del servicio (por ejemplo, nivel de precio contra calidad contra personal amistoso contra nivel de personalización), dependiendo de la clase de servicio que se evalúa y la importancia del mismo.

- Emociones de los clientes. Las experiencias propias del consumo pueden provocar emociones específicas que influyen en la satisfacción del cliente respecto del servicio.

- Atribuciones del fracaso o éxito del servicio. La atribución, es decir, las causas de los acontecimientos, también influye en las percepciones de satisfacción. Cuando se sorprende a los clientes con los resultados (ya sea que el servicio sea mejor o peor de lo esperado), ellos se inclinan a buscar las causas y la valoración que puede afectar su satisfacción.

- Percepciones de equidad o de justicia. Las nociones de justicia adquieren importancia central respecto a la percepción de satisfacción de los clientes en relación con los productos y servicios.

La satisfacción viene derivada tras una evaluación posterior a la compra de un producto y en función de las expectativas creadas con carácter previo a dicha compra; es así como definen la satisfacción del cliente como "el nivel del estado de ánimo" de una persona que resulta de comparar el rendimiento percibido de un producto o servicio con sus expectativas.

Para Zeithaml y colaboradores (2009), la satisfacción del cliente se identifica en la evaluación que da a un producto o servicio en función de si el mismo ha cumplido sus necesidades y expectativas. Para Hoffman y Bateson (2008), la sa- 
tisfacción del cliente proporciona una serie de beneficios para el proveedor del producto o servicio porque permite crear lealtad del cliente hacia la empresa, donde el consumidor estaría dispuesto a pagar más y a permanecer con la empresa si la misma satisface sus necesidades. Mientras que una insatisfacción del cliente puede, a través de la llamada comunicación de boca en boca, multiplicar por diez la mala publicidad que recibirá la empresa sin que esta pueda contrarrestarlo en un corto plazo, lo que lleva a pérdida de clientes y a tener mala imagen para con los actuales y futuros clientes.

\section{RESULTADOS DEL ESTUDIO}

Los resultados mostrados en la tabla 1, indican que de 271 entrevistados en los cuatro programas de postgrado, 178 son del sexo femenino y 93 masculinos. Del grupo de mujeres que cursan la Maestría Gerencia de Empresas, la mayoría se ubica entre 26 y 35 años de edad y la mayor parte de los hombres entre 35 y 50 años. Notándose en los otros programas, que los participantes se ubican en su mayoría, en las edades comprendidas entre los 26 y 50 años de edad.

Sin embargo, en la presente investigación se determina un predominio del sexo femenino en un $65,68 \%$, lo cual no coincide con los señalamientos de Gorrochotegui y otros (2006), (al menos para los Programas de Postgrado estudiados), donde determinan un crecimiento constante en la matrícula del sexo masculino y un estancamiento en la matrícula femenina.

Tabla 1. Características de la muestra. Edad y Género

\begin{tabular}{|l|c|c|c|c|c|c|c|c|}
\hline \multirow{2}{*}{ Programas } & \multicolumn{7}{c|}{ Edad/Género } \\
\cline { 2 - 10 } & \multicolumn{2}{c|}{$\begin{array}{c}\text { Menos de } \\
\mathbf{2 5} \text { años }\end{array}$} & \multicolumn{2}{c|}{$\begin{array}{c}26-35 \\
\text { años }\end{array}$} & \multicolumn{2}{c|}{$\begin{array}{c}\mathbf{3}-\mathbf{5 0} \\
\text { años }\end{array}$} & \multicolumn{2}{c|}{$\begin{array}{c}\text { Más de } \\
\mathbf{5 0} \text { años }\end{array}$} \\
\cline { 2 - 10 } & F & M & F & M & F & M & F & M \\
\hline $\begin{array}{l}\text { Doctorado en Ciencias Sociales: Mención } \\
\text { Gerencia }\end{array}$ & - & - & 2 & 4 & 4 & 12 & 4 & 13 \\
\hline Maestría en Gerencia de Empresas & 22 & 12 & 61 & 31 & 47 & 34 & 17 & 11 \\
\hline Maestría en Gerencia Pública & - & - & 9 & 15 & 3 & 11 & - & - \\
\hline Especialidad en Empresas Turísticas & - & - & 3 & 6 & 4 & 9 & 2 & 4 \\
\hline
\end{tabular}

Fuente: Elaboración propia

En la tabla 2, se detallan los resultados por programa de postgrado y se resumen los resultados de las razones para la selección de la Institución para cursar estudios. Se observa que el 30,5\% de los encuestados cursan estudios por el prestigio de la Institución dentro del sector universitario; el 22,5\%, señaló que preferían darle continuidad a sus estudios de cuarto y quinto nivel de estudios dentro de la misma institución; el 17,8\% opina que es menor la inversión comparada con las instituciones de carácter privado. El resto manifestó que la decisión fue tomada motivado por las exigencias laborales $(12,5 \%)$ y por decisión propia $(16,7 \%)$. 
Dianela Zuleta, Caterina Clemenza y Rubén Araujo

Calidad de servicio percibida por los usuarios de los programas de

postgrado en gerencia de la facultad de ciencias económicas y sociales

Tabla 2. Motivos para realizar estudios en la Institución

\begin{tabular}{|c|c|c|c|c|c|}
\hline & \multicolumn{4}{|c|}{ Programas } & \\
\cline { 2 - 5 } Aseveraciones & $\begin{array}{c}\text { Doctorado Ciencias } \\
\text { Sociales: Mención } \\
\text { Gerencia }\end{array}$ & $\begin{array}{c}\text { Maestría } \\
\text { Gerencia de } \\
\text { Empresas }\end{array}$ & $\begin{array}{c}\text { Maestría } \\
\text { Gerencia } \\
\text { Pública }\end{array}$ & $\begin{array}{c}\text { Especialidad } \\
\text { Empresas } \\
\text { Turísticas }\end{array}$ & $\begin{array}{c}\text { Promedio } \\
(\%)\end{array}$ \\
\hline $\begin{array}{c}\text { Prestigio de la } \\
\text { institución }\end{array}$ & $27 \%$. & $42 \%$ & $31 \%$ & $22 \%$ & 30,5 \\
\hline $\begin{array}{c}\text { Costos de } \\
\text { inversión }\end{array}$ & $19 \%$ & $6 \%$ & $13 \%$ & $33 \%$ & 17,8 \\
\hline $\begin{array}{c}\text { Continuidad de } \\
\text { estudios dentro } \\
\text { de la misma } \\
\text { institución }\end{array}$ & $27 \%$ & $33 \%$ & $18 \%$ & $12 \%$ & 22,5 \\
\hline $\begin{array}{c}\text { Exigencias } \\
\text { laborales }\end{array}$ & $9 \%$ & $5 \%$ & $25 \%$ & $11 \%$ & 12,5 \\
\hline Decisión propia & $18 \%$ & $14 \%$ & $13 \%$ & $22 \%$ & 16,7 \\
\hline
\end{tabular}

Fuente: Elaboración propia

En primer lugar se analizan los valores medios de las expectativas y percepción de los clientes de todos los atributos que caracterizan la calidad del servicio, para la cual se establece detallándose la diferencia entre percepciones y expectativas (P-E).

En cuanto a las expectativas, se observa un valor medio de 4.53, para los elementos tangibles; 4,74 para la fiabilidad; 4,62 para la capacidad de respuesta, 4,75 para la seguridad y 4,68 para la empatía, todo lo cual asciende a una media global de 4,66 confirmando el nivel de exigencias de los participantes que cursan estos programas de postgrado.

$\mathrm{Al}$ detenernos en las percepciones de los clientes se tiene un 3,17 para los elementos tangibles; 3,82 para la fiabilidad; 3,39 para la capacidad de respuesta; 3,62 para la seguridad y 3,63 para la empatía; siendo 3,17 la media correspondiente a los componentes de la calidad percibida por los estudiantes entrevistados.

Al establecer la diferencia entre los valores medios dados en la percepción y la expectativa (P-E) da como resultado un valor negativo de $-1,36$ lo cual refleja que los servicios ofrecidos deben ser mejorados para lograr satisfacer a los clientes.

En segundo lugar, al detallar en el análisis para lo cual se establecen tres categorías (alta, media y baja) para los atributos de la calidad del servicio (Tabla 3), se observa que las categorías con niveles de expectativa alta se corresponden a los atributos relacionados con los aspectos tangibles e intangibles (fiabilidad, capacidad de respuesta seguridad y empatía).

En tanto que los atributos tangibles e intangibles son percibidos como medios, a excepción de la disponibilidad del servicio de reproducción de material impreso $(2,57)$ que ésta presenta baja percepción. 
Tabla 3. Categorías para las Expectativas y la Percepción

\begin{tabular}{|c|c|}
\hline Rango & Expectativas/Percepción \\
\hline $1,00-1,80$ & \multirow{2}{*}{ Baja } \\
\hline $1,81-2,60$ & \\
\hline $2,61-3,40$ & \multirow{2}{*}{ Media } \\
\hline $3,41-4,20$ & \\
\hline $4,21-5,00$ & Alta \\
\hline
\end{tabular}

Fuente: Elaboración propia

Por último, al analizar las diferencias entre expectativas y percepciones (E-P), se debe hacer hincapié en ninguno de los atributos, las percepciones exceden las expectativas, arrojando una brecha negativa. Se observan además, que dentro del grupo de resultados negativos, existen algunas con valores reducidos por debajo de la media.

En cuanto a los atributos que presentan los valores negativos más altos, se encuentran en los aspectos tangibles como: Horario de atención de las oficinas administrativas ajustado a las necesidades de los estudiantes $(-1,79)$; la disponibilidad del servicio de reproducción $(-1,89)$, Respecto a la seguridad, la protección de su vehículo en el estacionamiento de la institución $(-1,74)$ y seguridad personal dentro de las instalaciones de la institución $(-1,49)$. Llama la atención, dentro del atributo empatía, como el trato respetuoso y cortés del personal administrativo al atender las necesidades de los estudiantes muestra una brecha de -1,41.

Los datos relacionados a la medición de expectativas y percepciones de los clientes respecto a la calidad de servicio y a la satisfacción de los usuarios de los programas de postgrado en Gerencia, de la Facultad de Ciencias Económicas y Sociales de la Universidad del Zulia, se observan en la tabla 4.

Tabla 4. Brecha entre las Expectativas y la Percepción

\begin{tabular}{|l|c|c|c|}
\hline \multicolumn{1}{|c|}{ Subdimensiones e Indicadores } & Percepción & Expectativas & Brecha \\
\hline \multicolumn{1}{|c|}{ Elementos Tangibles } & 3.17 & 4.53 & -1.36 \\
\hline (a) Apariencia de las instalaciones & & & \\
\hline Existencia de confort en las aulas de clase & 3.62 & 4.52 & -0.90 \\
\hline $\begin{array}{l}\text { Buenas condiciones de las salas sanitarias de la insti- } \\
\text { tución }\end{array}$ & 3.43 & 4.70 & -1.27 \\
\hline $\begin{array}{l}\text { Facilidad de acceso al estacionamiento de la institu- } \\
\text { ción }\end{array}$ & 3.59 & 4.44 & -0.85 \\
\hline \multicolumn{1}{|c|}{ (b) Horario de Atención } & & & \\
\hline $\begin{array}{l}\text { Horario de atención de las oficinas administrativas } \\
\text { ajustado a las necesidades de los estudiantes }\end{array}$ & 2.82 & 4.61 & -1.79 \\
\hline $\begin{array}{l}\text { Horario de atención de la biblioteca ajustado a las ne- } \\
\text { cesidades de los estudiantes }\end{array}$ & 3.24 & 4.61 & -1.37 \\
\hline
\end{tabular}


Dianela Zuleta, Caterina Clemenza y Rubén Araujo

Calidad de servicio percibida por los usuarios de los programas de

postgrado en gerencia de la facultad de ciencias económicas y sociales

\begin{tabular}{|c|c|c|c|}
\hline Subdimensiones e Indicadores & Percepción & Expectativas & Brecha \\
\hline \multicolumn{4}{|l|}{ (c) Recursos tecnológicos y materiales } \\
\hline $\begin{array}{l}\text { La disponibilidad de equipos computarizados sufi- } \\
\text { cientes para la realización de las labores del personal } \\
\text { dentro de la institución }\end{array}$ & 2.91 & 4.43 & -1.52 \\
\hline $\begin{array}{l}\text { La disponibilidad de equipos computarizados actua- } \\
\text { lizados y en buenas condiciones para la realización de } \\
\text { las labores del personal dentro de la institución }\end{array}$ & 2.84 & 4.45 & -1.61 \\
\hline $\begin{array}{l}\text { La disponibilidad del servicio de reproducción cuan- } \\
\text { do lo requiere el estudiante }\end{array}$ & 2.57 & 4.46 & -1.89 \\
\hline $\begin{array}{l}\text { La disponibilidad de textos actualizados en la biblio- } \\
\text { teca }\end{array}$ & 3.08 & 4.67 & -1.59 \\
\hline La disponibilidad de textos variados en la biblioteca & 3.12 & 4.60 & -1.48 \\
\hline \multicolumn{4}{|l|}{ (d) Apariencia del Personal } \\
\hline Personal de aspecto cuidado & 3.36 & 4.32 & -0.96 \\
\hline $\begin{array}{l}\text { Identificación del personal según las funciones que } \\
\text { realiza dentro de la institución }\end{array}$ & 3.46 & 4.57 & -1.11 \\
\hline $\begin{array}{l}\text { Fiabilidad } \\
\end{array}$ & 3.82 & 4.74 & -0.92 \\
\hline $\begin{array}{l}\text { Cumplimiento de los deberes por parte del personal } \\
\text { de la institución }\end{array}$ & 3.55 & 4.56 & -1.01 \\
\hline $\begin{array}{l}\text { Cumplimiento del horario establecido por parte del } \\
\text { personal de la institución }\end{array}$ & 3.44 & 4.58 & -1.14 \\
\hline $\begin{array}{l}\text { Habilidad de los profesores para transmitir conoci- } \\
\text { mientos }\end{array}$ & 4.10 & 4.90 & -0.80 \\
\hline $\begin{array}{l}\text { Conocimiento actualizado del profesor sobre la cáte- } \\
\text { dra que dicta }\end{array}$ & 4.18 & 4.92 & -0.73 \\
\hline Capacidad de respuesta & 3.39 & 4.62 & -1.23 \\
\hline $\begin{array}{l}\text { Información oportuna sobre los servicios prestados } \\
\text { por la institución }\end{array}$ & 3.44 & 4.63 & -1.19 \\
\hline $\begin{array}{l}\text { Procesamiento oportuno de las solicitudes realizadas } \\
\text { por los estudiantes }\end{array}$ & 3.44 & 4.65 & -1.21 \\
\hline $\begin{array}{l}\text { Prontitud del personal para responder ante imprevis- } \\
\text { tos }\end{array}$ & 3.36 & 4.65 & -1.29 \\
\hline $\begin{array}{l}\text { Operación continua de los programas computariza- } \\
\text { dos de control de estudios }\end{array}$ & 3.33 & 4.56 & -1.22 \\
\hline Seguridad & 3.62 & 4.75 & -1.13 \\
\hline $\begin{array}{l}\text { Relación del costo de la matrícula con el servicio pres- } \\
\text { tado }\end{array}$ & 3.78 & 4.73 & -0.95 \\
\hline $\begin{array}{l}\text { Aplicación del conocimiento obtenido en el transcur- } \\
\text { so de los estudios del postgrado en su área laboral }\end{array}$ & 4.11 & 4.75 & -0.63 \\
\hline Seguridad al realizar transacciones con la institución & 3.85 & 4.71 & -0.86 \\
\hline $\begin{array}{l}\text { Seguridad personal dentro de las instalaciones de la } \\
\text { institución }\end{array}$ & 3.30 & 4.80 & -1.49 \\
\hline
\end{tabular}




\begin{tabular}{|c|c|c|c|}
\hline Subdimensiones e Indicadores & Percepción & Expectativas & Brecha \\
\hline $\begin{array}{l}\text { Seguridad en cuanto a la protección de su vehículo en } \\
\text { el estacionamiento de la institución }\end{array}$ & 3.03 & 4.77 & -1.74 \\
\hline Empatía & 3.63 & 4.68 & -1.05 \\
\hline $\begin{array}{l}\text { Trato respetuoso y cortés del personal administrativo } \\
\text { al atender las necesidades de los estudiantes }\end{array}$ & 3.28 & 4.69 & -1.41 \\
\hline $\begin{array}{l}\text { Disposición del personal de la biblioteca para atender } \\
\text { las necesidades de los estudiantes }\end{array}$ & 3.67 & 4.66 & -0.99 \\
\hline $\begin{array}{l}\text { Facilidad en la comunicación entre el personal admi- } \\
\text { nistrativo y los estudiantes }\end{array}$ & 3.44 & 4.57 & -1.13 \\
\hline $\begin{array}{l}\text { Atención personalizada de los profesores hacia los } \\
\text { estudiantes }\end{array}$ & 4.08 & 4.80 & -0.73 \\
\hline MEDIA & 3.51 & 4.66 & -1.15 \\
\hline
\end{tabular}

Fuente: Elaboración propia

Los niveles de expectativas y percepción se evaluaron en una escala comprendida entre $(1,0)$ y $(5,0)$ para señalar menor y mayor valoración, respectivamente. Por lo tanto, el nivel de satisfacción de los clientes o la brecha existente entre las puntuaciones entre percepción y expectativas de los clientes encuestados con respecto a los componentes de la calidad de servicios, estará comprendida en la escala $(-4,0)$ "Muy Insatisfecho" y $(4,0)$ "Muy Satisfecho" (Tabla 5). Los valores comprendidos entre $-0,8$ y 0,8 corresponden a niveles de satisfacción "Neutral" y se considera que el nivel percibido se corresponde con el nivel esperado $(\mathrm{P} \sim \mathrm{E})$.

De acuerdo a la tabla 6, un nivel de satisfacción “Un Poco Insatisfecho" equivale a un nivel de calidad de servicio baja

Tabla 5. Escala: Nivel de Satisfacción

\begin{tabular}{|l|c|c|c|}
\hline \multicolumn{1}{|c|}{ Nivel de Satisfacción } & \multicolumn{2}{|l}{} & $\begin{array}{c}\text { Calidad de } \\
\text { Servicio }\end{array}$ \\
\cline { 1 - 3 } Muy Satisfecho & 2,4 & 4,0 & \multirow{2}{*}{ Alta } \\
\hline Un Poco Satisfecho & 0,8 & 2,4 & \multirow{2}{*}{ Neutral } \\
\hline Neutral & $-0,8$ & 0,8 & \multirow{2}{*}{ Baja } \\
\cline { 1 - 3 } Un Poco Insatisfecho & $-2,4$ & $-0,8$ & \\
\cline { 1 - 3 } Muy Insatisfecho & $-4,0$ & $-2,4$ & \\
\hline
\end{tabular}

Fuente: Elaboración propia

Como comentario se tiene que, Zeithaml y Bitner (2002) señalan que la calidad de servicio percibida es el resultado de comparar las percepciones del cliente sobre el nivel de servicio prestado por una organización, y las expectativas sobre el nivel de prestación que se debería esperar de esa categoría de servicio. Se tiene, entonces 
que los participantes de los programas de postgrado en gerencia, estudiados manifestaron poseer expectativas superiores a sus percepciones, basadas en un conjunto de atributos, considerados como los indicadores de las únicas dimensiones básicas que desfavorecen la calidad del servicio.

Tabla 6. Nivel de Satisfacción

\begin{tabular}{|c|c|c|c|c|c|}
\hline Sub - Dimensiones & Percepción & Expectativas & Brecha & $\begin{array}{c}\text { Calidad de } \\
\text { Servicio }\end{array}$ & $\begin{array}{c}\text { Nivel de } \\
\text { satisfacción }\end{array}$ \\
\hline Tangibilidad & 3.17 & 4.53 & -1.36 & Baja & $\begin{array}{c}\text { Un poco } \\
\text { insatisfecho }\end{array}$ \\
\hline Fiabilidad & 3.82 & 4.74 & -0.92 & Baja & $\begin{array}{c}\text { Un poco } \\
\text { insatisfecho }\end{array}$ \\
\hline $\begin{array}{c}\text { Capacidad de } \\
\text { Respuesta }\end{array}$ & 3.39 & 4.62 & -1.23 & Baja & $\begin{array}{c}\text { Un poco } \\
\text { insatisfecho }\end{array}$ \\
\hline Seguridad & 3.62 & 4.75 & -1.13 & Baja & $\begin{array}{c}\text { Un poco } \\
\text { insatisfecho }\end{array}$ \\
\hline Empatía & 3.63 & 4.68 & -1.05 & Baja & $\begin{array}{c}\text { Un poco } \\
\text { insatisfecho }\end{array}$ \\
\hline Promedio & 3.51 & 4.66 & -1.15 & Baja & $\begin{array}{c}\text { Un poco } \\
\text { insatisfecho }\end{array}$ \\
\hline
\end{tabular}

Fuente: Elaboración propia

Si a esta consideración, se le agrega que mientras más alta o baja sea la calidad, percibida; es decir, mientras menos o más desviación haya entre la diferencia de percepciones y expectativas, el cliente se sentirá satisfecho o insatisfecho, Berry (2004); Hoffman y Bateson (2002); Zeithaml y Bitner (2002); se puede señalar, de acuerdo a los resultados, que el servicio percibido no satisface a los estudiantes.

Normalmente el servicio que recibe el cliente es el resultado de una cadena de servicios interrelacionados, donde no sólo las evidencias físicas del mismo son evaluadas por los clientes, sino que además contemplan el carácter intangible de los servicios. Según Stanton, Etzel y Walter (2004), es posible aplicar cuatro estrategias a fin de reducir el impacto que produce sobre el cliente. La primera de ellas es la visualización, que consiste en ilustrar los beneficios del servicio.

\section{CONSIDERACIONES FINALES}

De acuerdo a los planteamientos expresados anteriormente, se puede concluir que los participantes de los programas de postgrado en Gerencia de la División de Estudios para Graduados de la Facultad de Ciencias Económicas y Sociales de la Universidad del Zulia, perciben que la calidad de servicio cubre medianamente sus expectativas en cuanto a los cinco componentes analizados (tangibilidad, fiabilidad, capacidad de respuesta, seguridad y empatía), lo cual lleva a determinar que se encuentran un poco insatisfechos. 
Esto hace suponer que la institución ofrece un servicio de calidad aceptable; pero que los clientes esperan un mejor desempeño, logrando así ajustarse a sus altas exigencias.

Se hace necesario emprender acciones que estén dirigidas a mejorar la percepción de los participantes en cuanto a los atributos intangibles que presentaron valores negativos más altos, como la seguridad y la empatía. Así como fortalecer los elementos físicos y el ambiente de la organización, debido a que una percepción positiva de ellos puede tener un efecto sobre la imagen que los participantes se hagan del lugar. En este sentido, estos elementos pueden ser vistos como una forma de comunicación no verbal que confiere significado a la calidad percibida.

\section{REFERENCIAS}

ALBRECHT, Karl y ZENKE, Ron (1998). Gerencia de Servicio. Séptima Edición. Editorial Legis, Bogotá, Colombia.

BERRY, Leonard (2004). Un buen servicio ya no basta. Ediciones Deusto, España.

GORROCHOTEGUI, Alfredo; MARIÑO, Edison y TENIAS, Virginia (2006). Educación superior privada en Venezuela. IESAL/Unesco. http://www.unesco.org.ve, Caracas, Venezuela.

HOFFMAN, K. Douglas; BATESON, John (2002). Fundamentos de Marketing de Servicios. Conceptos, estrategias y casos. Segunda Edición. Editorial Thomson, México.

MORLES, Víctor; MEDINA, Eduardo y ÁLVAREZ, Neptali (2003). La educación superior en Venezuela. IESAL/ Unesco. http://www.unesco.org.ve, Caracas, Venezuela.

STANTON, W.; ETZEL, M.;WALTER, B. (2004). Fundamentos de Marketing. Mc Graw-Hill, México, 13ª Edición.

ZEITHAML, Valarie y BITNER, Mary Jo (2002). Marketing de servicios. Editorial McGraw-Hill Interamericana, México, segunda edición.

ZEITHAML, Valarie; BITNER, Mary Jo y GREMLER, Dwayne (2009). Marketing de servicios. Editorial McGrawHill Interamericana, México, quinta edición. 